\title{
UMA ABORDAGEM SOCIOMÉTRICA PARA A REDE DE EMPREENDEDORISMO
}

\author{
Valquirio Jóia Marques da Silva Filho ${ }^{1}$ \& André Fernando Uebe Mansur ${ }^{2 *}$
}

\begin{abstract}
RESUMO
SILVA FILHO, V.J.M.,UEBE MANSUR,A.F. Uma Abordagem Sociométrica para a Rede de Empreendedorismo. Perpectivas Online: Humanas \& Sociais Aplicadas, v.7, n.19, p.31-38, 2017.
\end{abstract}

O desenvolvimento regional passa, inevitavelmente, pela articulação dos atores econômicos e demais stakeholders de uma região. A existência de canais para diálogo e interlocução dos mesmos fortalece e amplia as possibilidades de incremento econômico e desenvolvimento, principalmente nos setores relacionados às Pequenas e Médias empresas (PMEs). Buscando explorar os recursos tecnológicas que permitem vínculo em rede destes atores, os papéis de instituições de fomento, como incubadoras de empresa e instituições de ensino, se intensificam, uma vez que estas veem ampliadas seu poder de articulação para além das fronteiras físicas regionais de atuação. A possibilidade de se fazer uso de Novas Tecnologias da Informação e Comunicação (NTICs) se mostram como uma possibilidade mais que viável para o fomento ao desenvolvimento regional e acadêmico, dada pela associação de indivíduos com ideias criativas e organizações empreendedoras. Buscando uma articulação entre pesquisa e extensão, a Join Venture Social Network (JoVen) se vislumbra, portanto, como um projeto focado no uso de tecnologias open source e recursos que promovam portabilidade e mobilidade, por meio de uma Rede Social Digital nos campos da Administração, Economia e Tecnologia, criando um ambiente digital que permita a conexão e diálogo entre proponentes de projetos empreendedores e propensos investidores. Este projeto objetiva facilitar a conexão e diálogos entre proponentes empreendedores e propensos investidores. Para colocar em pratica ideias e projetos de empreendedorismo, em realidade, através de uma rede virtual digital. Até o presente momento, alguns passos significativos podem ser apresentados, como: definição do tipo de rede digital que será usado, telas e sistemas de requisitos do sistema.

Palavras-chave: Empreendedores; Rede digital; Investidores. 


\section{ABSTRACT}

The regional development inevitably involves the articulation of economic actors and other stakeholders in a region. The existence of channels for dialogue among them strengthens and broadens the possibilities of economic growth and development, especially in the sectors related to Small and Medium Enterprises (SMEs). In order to exploit the technological resources that allow these actors to be networked, the roles of development institutions, such as business incubators and educational institutions, intensify, once they have expanded their power of articulation beyond regional physical boundaries. The possibility of making use of Information and Communication Technologies (ICTs) are shown as a viable possibility for the promotion of regional and academic development, given by the association of individuals with creative ideas and entrepreneurial organizations. Seeking a link between research and extension, the Join Venture Social Network (JoVen) is therefore seen as a project focused on the use of open source technologies and resources that promote portability and mobility, through of a Digital Social Network in the fields of Administration, Economy and Technology, creating a digital environment that allows the connection and dialogue between proponents of entrepreneurial projects and prone investors. This project aims to facilitate the connection and dialogues between entrepreneurial proponents and prospective investors. To put into practice ideas and projects of entrepreneurship, in reality, through a digital channel. To date, some significant steps have been made as definition of the type of digital network that will be used, screens and software systems defined.

Keywords: Entrepreneurs; Digital network; Investors.

\footnotetext{
${ }^{1}$ Acadêmico em Administração ISECENSA- Institutos Superiores de Ensino do CENSA - ISECENSA - Rua Salvador Correa, 139, Centro, Campos dos Goytacazes, RJ, CEP: 28035-310, Brasil;

${ }^{2}$ Instituto Federal de Educação, Ciência e Tecnologia Fluminense- IFF- Rua Dr. Siqueira, 273 - Parque Dom Bosco Campos dos Goytacazes, RJ, CEP 28030-130 - ISECENSA - Laboratório Adm. Empresas e Sistemas de ComputaçãoRua Salvador Correa, 139, Centro, Campos dos Goytacazes, RJ, CEP: 28035-310, Brasil.

(*)e-mail: andreuebe@gmail.com

Data de chegada: 19/04/2017 Aceito para publicação: 09/06/2017
} 


\section{INTRODUÇ̃̃̃O}

Conforme Carvalho e Totti (2006), o desenvolvimento econômico da região Norte Fluminense, significativamente representada pelo município de Campos dos Goytacazes, teve a indústria sucroalcooleira e a agropecuária como principais atividades. Com a perda do potencial produtivo em ambas os setores, assim como o início da exploração petrolífera de na Bacia de Campos, em 1977, este cenário econômico começou a se diversificar.

O início das atividades da Universidade Estadual do Norte Fluminense Darcy Ribeiro (UENF), em 1993 representaram uma diversificação alternativa e definitiva ao cenário econômico regional, uma vez que a partir deste momento, começou a se consolidar o processo de transformação do município de Campos dos Goytacazes em um pólo universitário (UENF,2014). Atualmente a cidade de Campos dos Goytacazes se caracteriza como um pólo universitário com a presença de 12 universidades, sendo destas, três públicas.

Destas instituições públicas de ensino superior, destaca-se o Instituto Federal de Educação Ciência e Tecnologia Fluminense (IF Fluminense), uma das 38 unidades de ensino criadas pelo Governo Federal em 2008, a partir dos Centros Federais de Educação Tecnológica e das Escolas Agrotécnicas, como parte do plano de expansão da Rede Federal de ensino, iniciado em 2005. (IF Fluminense, 2014). Apesar da região se caracterizar como um polo universitário e expressar um significativo desenvolvimento econômico por novos arranjos produtivos relacionados com o petróleo, a logística e a educação, algumas problemáticas sociais se evidenciam e se agravam.

Uma destas questões se refere à desarticulação de diversos setores produtivos sociais como, por exemplo, a indústria e a educação. Esta desarticulação se manifesta, por exemplo, pela falta de um efetivo diálogo entre a indústria e os centros de formação profissionais técnicos, tecnológicos e universitários, levando à situações como:

- Inadequado repasse por parte das instituições industriais, assim como inadequada captação por parte das instituições acadêmicas, das possibilidades de parcerias que poderiam promover novas soluções aos problemas sociais;

- A formação de profissionais que não atendem plenamente aos quesitos de habilidades técnicas demandas pelos setores produtivos locais, levando ao contrassenso de importação de mão de obra em um polo universitário;

- Insatisfatório aproveitamento do potencial de soluções inovadoras aos problemas produtivos, por parte das instituições acadêmicas;

- Atuação periférica, por parte do poder público municipal, em ações estratégicas que possibilitariam um investimento efetivo em programas de geração de emprego e renda;

Em consonância com estas problemáticas, Marteleto e Silva (2004) discutem importância do uso de metodologias relacionadas a análise de redes sociais para a compreensão do acesso à informação e as consequentes resultantes deste fenômeno no desenvolvimento econômico social. No estudo em questão os autores apresentam os estudos de Granovetter (1973) que coloca as redes interpessoais como elemento fundamental na ponte que se estabelece entre dois indivíduos distintos e não conectados e que contribuem para as transformações histórico sociais.

Neste estudo, é analisado o fato de que as relações fortes (estabelecida entre indivíduos que já possuem uma relação) não contribuem tão significativamente para a ampliação dos limites das redes quanto o fortalecimento de relação fracas (existente entre indivíduos cujo o envolvimento emocional é originalmente menor ou nulo). Marteleto e Silva (2004) concluem em seus estudos que o acesso à informação, incrementado pelo uso de redes sociais, é de grande importância para o desenvolvimento de empresas e comunidades, por propiciarem acesso à informação e estabelecimento de pontes previamente inexistentes pelo fortalecimento de relações originalmente fracas. O desenvolvimento e uso intensivo de metodologias e ferramentas computacionais buscam facilitar a realização de trabalhos colaborativos entre grupos com interesses comuns, permitindo que a ciência evolua cada vez mais rápido, dado o compartilhamento de dados, ideias e experimentos em tempo real (MARCUS, 2008).

Um exemplo destas ferramentas é o aplicativo DiscoVR disponível para o sistema IOS (conforme figura 1), que traz conexões relacionadas a projetos na área de música, mas cuja ideia é perfeitamente adaptável a uma Rede Social Digital (RSD) para empreendedorismo. 


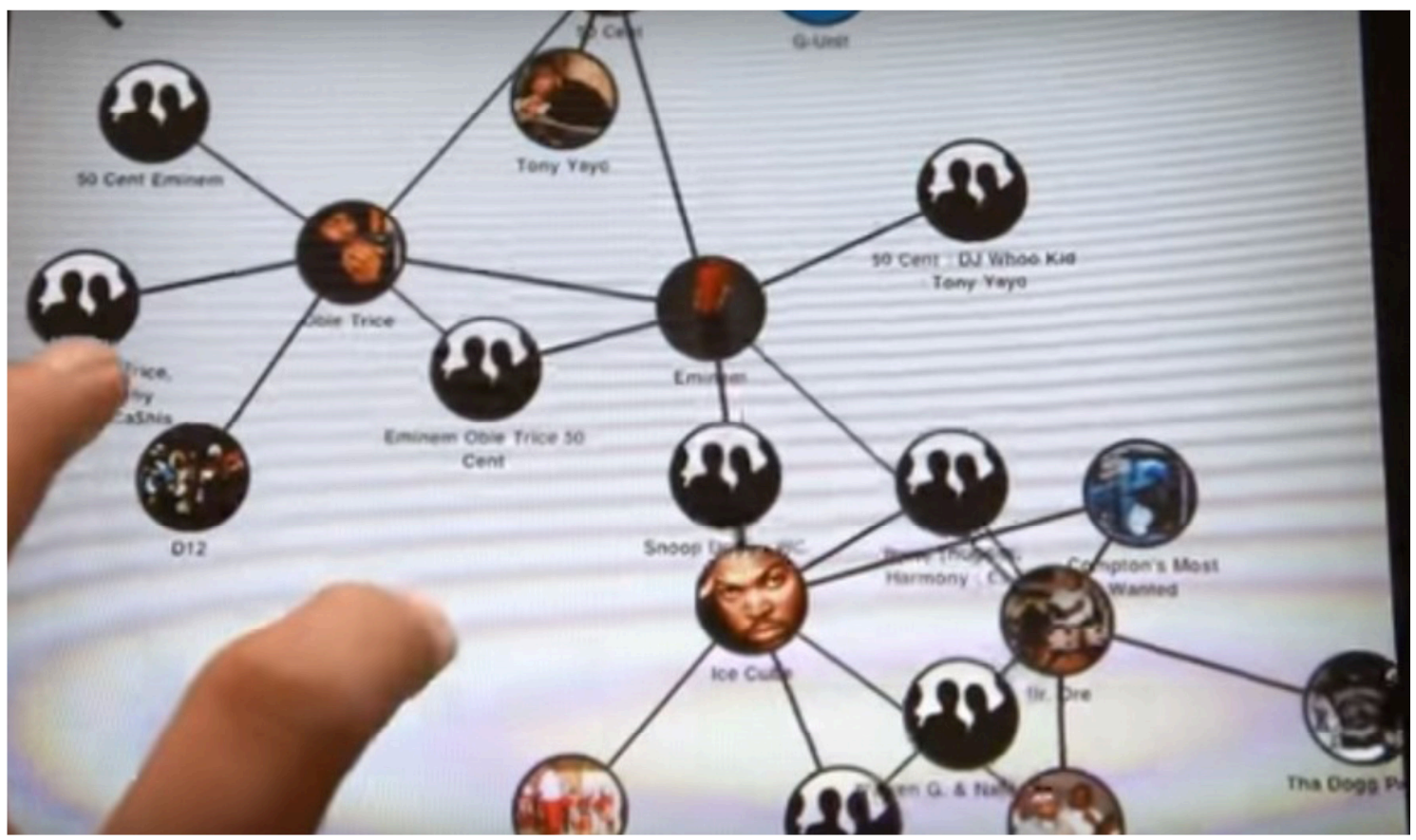

Figura 1: DiscoVR - Rede Social rizomática de música.

Assim, surge a hipótese de que ambientes complexos como os das Redes Sociais Digitais (RSD) podem desempenhar um significativo papel na articulação destes atores sócio econômicos e contribuir para o bem-estar social.

Por esta razão, justificam-se novas propostas, como o presente projeto, que incentivam a criação de ambientes compartilhados por atores sócio econômicos, removendo as barreiras impostas pelo espaço físico e tempo, como pressuposto para definição a estratégias que levem a um melhor equilíbrio social na região, pelo incremento ao empreendedorismo. Recentemente trabalho correlato foi publicado por UEBE MANSUR (2015) et al corroborando o uso de uma abordagem sociométrica como abordagem teórica de desenvolvimento do ambiente proposto, o que aponta a presente pesquisa, também, para a vertente acadêmica haja vista a possibilidade de fomento por meio de estudo e prática, do tema empreendedorismo no contexto educacional.

Com o intuito de desenvolver um protótipo de uma rede digital que possibilite conectar promover diálogo entre atores econômicos (indivíduos que possuam alguma ideia empreendedora e que necessitem do apoio de investidores). Com benefício a sociedade, a efetividade de um ambiente digital em rede social que permite a interação entre atores econômicos voltados ao empreendedorismo, possibilita um real incentivo ao desenvolvimento local e regional, fomentando a economia e o surgimento de novos negócios. Esta ideia é respaldada por Barabási (2002) que afirma ser possível fortalecer as conexões fracas de uma rede e promover a criação de cluster que geram um efeito cascata de crescimento de conexões e fenômenos relacionados a esta rede. Proteção da Propriedade Intelectual (quando cabível).

Os aprimoramentos tecnológicos decorrentes deste projeto enquadram-se na categoria de autoria de Creative Common e se efetivará pela publicação dos resultados em eventos científicos das áreas correlatas. Ademais há, ainda a possibilidade de registro de patentes por meio do Núcleo de Inovação Tecnológica. Objetivos Gerais:

- Estudo e desenvolvimento de ambiente digital, baseado nos conceitos de Redes Sociais Digitais (RSD), que promova a interlocução entre proponentes de projetos empreendedores e propensos investidores.

Objetivos Específicos:

- Promover a cultura empreendedora, assim como sua prática no ambiente acadêmico; 
- Buscar interlocuções com centros de pesquisa e pesquisadores que possibilitem aprofundamentos no tema proposto;

- Propor a modelagem de um ambiente que favoreça à formação de redes e subredes de indivíduos com interesses complementares;

- Divulgar e incentivar o uso do ambiente compartilhado proposto e avaliar o impacto do mesmo na sinergia dos grupos dispersos em uma região e no desenvolvimento local e regional.

\section{METODOLOGIA}

Para o desenvolvimento do presente projeto, estabelece-se uma metodologia de busca de conhecimento teórico (conceitual) e empírico (casos reais) em centros de estudo onde o bem estar social e o desenvolvimento econômico são realidades bem mais plausíveis que no Brasil, para que seja possível o estudo e proposição de estratégias adaptadas à realidade local utilizando-se, prioritariamente, dos conceitos de Redes Sociais como promotoras do desenvolvimento econômico e bem estar social. Adquirindo conhecimentos através de:

- Artigos (Revista administração contemporânea, artigo sobre empreendedorismo sustentável);

- Livro (Analise de redes sociais, um livro que nos mostra o desenvolvimento das redes sociais e nos mostra amplamente como essa ferramenta pode ser benéfica para todas as pessoas);

- Site de namoro (Zoosk);

- Sites de anúncios (OLX) e;

- Aplicativos com propósitos de empreendedorismo e inovação (Minas Inova).

Foi definido reuniões semanais com os bolsistas do IFF e os orientadores para desenvolvimento do projeto, sendo que minhas pesquisas são pesquisas de campo e as pesquisas dos bolsistas do IFF são tecnológicas. Juntos testamos as duas ideias. Definido o uso da ferramenta Trello para comunicação e para postar avanços do projeto. Foi elaborado um cronograma com metas para desenvolvimento do projeto a cada mês. Neste contexto, as ações a serem desenvolvidas serão distribuídas em duas etapas com duração de 6 meses cada uma delas. As ações a serem realizadas na primeira etapa referem-se ao planejamento do projeto:

- Contatos e interações iniciais com os aspectos culturais e acadêmicos dos grupos de pesquisa relacionados ao projeto;

- Intercâmbio de conhecimentos e troca de experiências relacionadas as áreas de interesse com respectiva seleção dos temas mais correlatos a esta proposta;

- Verificação dos aspectos da realidade local em consonância aos percursos viáveis e alternativos definidos na etapa de planejamento;

- Levantamento de soluções tecnológicos atualmente existentes, correlatas ao tema;

- Estudo para possíveis adequações das soluções tecnológicas existentes à realidade operacional do projeto.

A segunda etapa, referente ao desenvolvimento do protótipo, se dá pelas seguintes ações:

- Definição das tecnologias Open Source a serem adotas para o desenvolvimento do projeto;

- Definição dos requisitos funcionais do protótipo;

- Modelagem do banco de dados;

- Implementação do protótipo;

- Implantação, testes e adequações do protótipo;

- Análise de resultados. 


\section{RESULTADOS E DISCUSSÕES}

O projeto JoVen já possui logotipo (conforme figura 1). Está sendo definido através de estudos qual o tipo de sistema será usado. Está sendo desenvolvida a página inicial, no qual contará com login, recuperação de senha e cadastro de usuário (conforme figura 2). A próxima página a ser definida e que está sendo estudada e planejada será a página de cadastro de perfil especifico, que após cadastro inicial simples, com e-mail e senha o usuário irá fazer um cadastro do seu perfil individual, ou seja, perfil empreendedor, perfil investidor, perfil capacitador intelectual e perfil incubadora (conforme figura 3). Estruturação da tela de perfil, com nome, local, vídeos, imagens e projetos salvos (conforme figura 4).
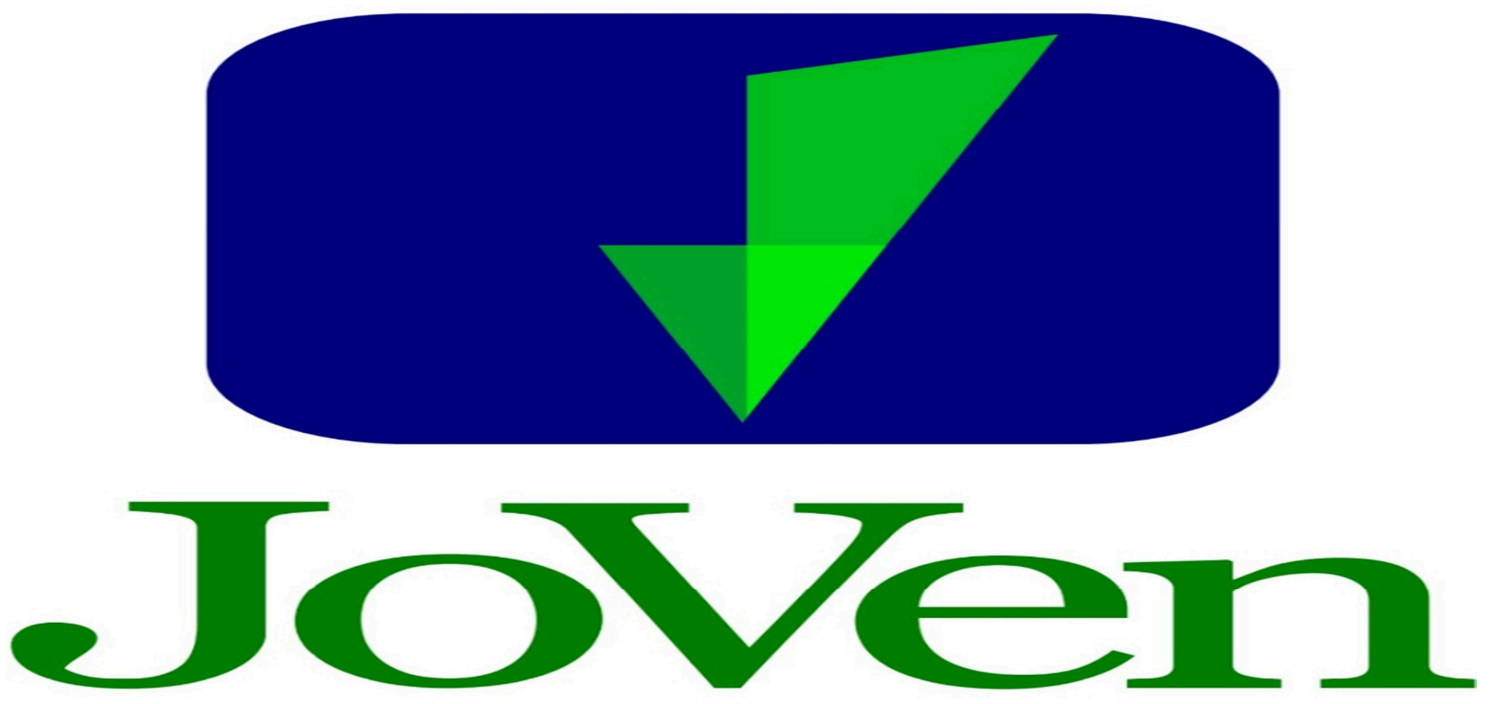

Figura 1: Logotipo projeto JoVen.

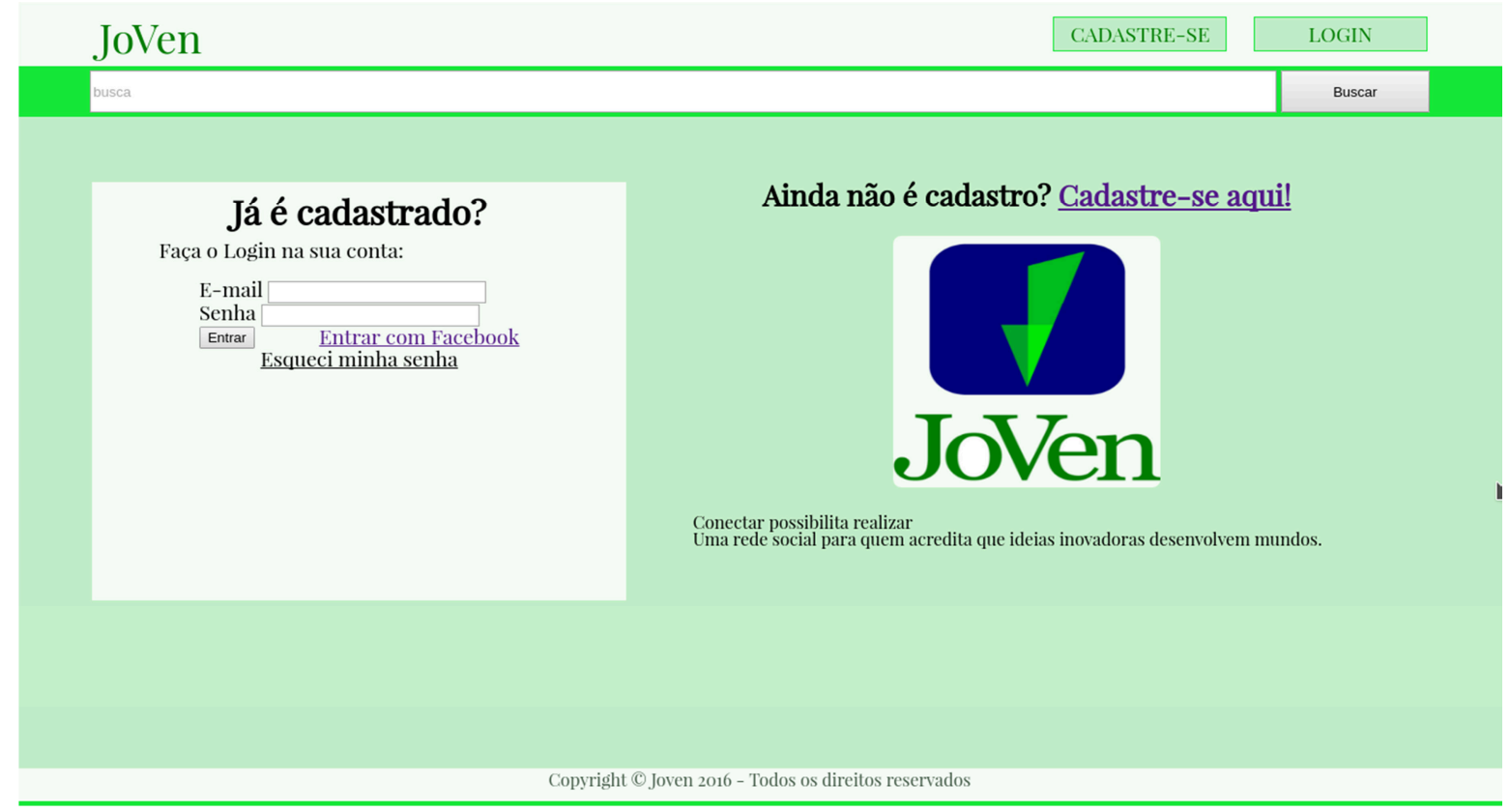

Figura 2: Tela de cadastro e acesso. 


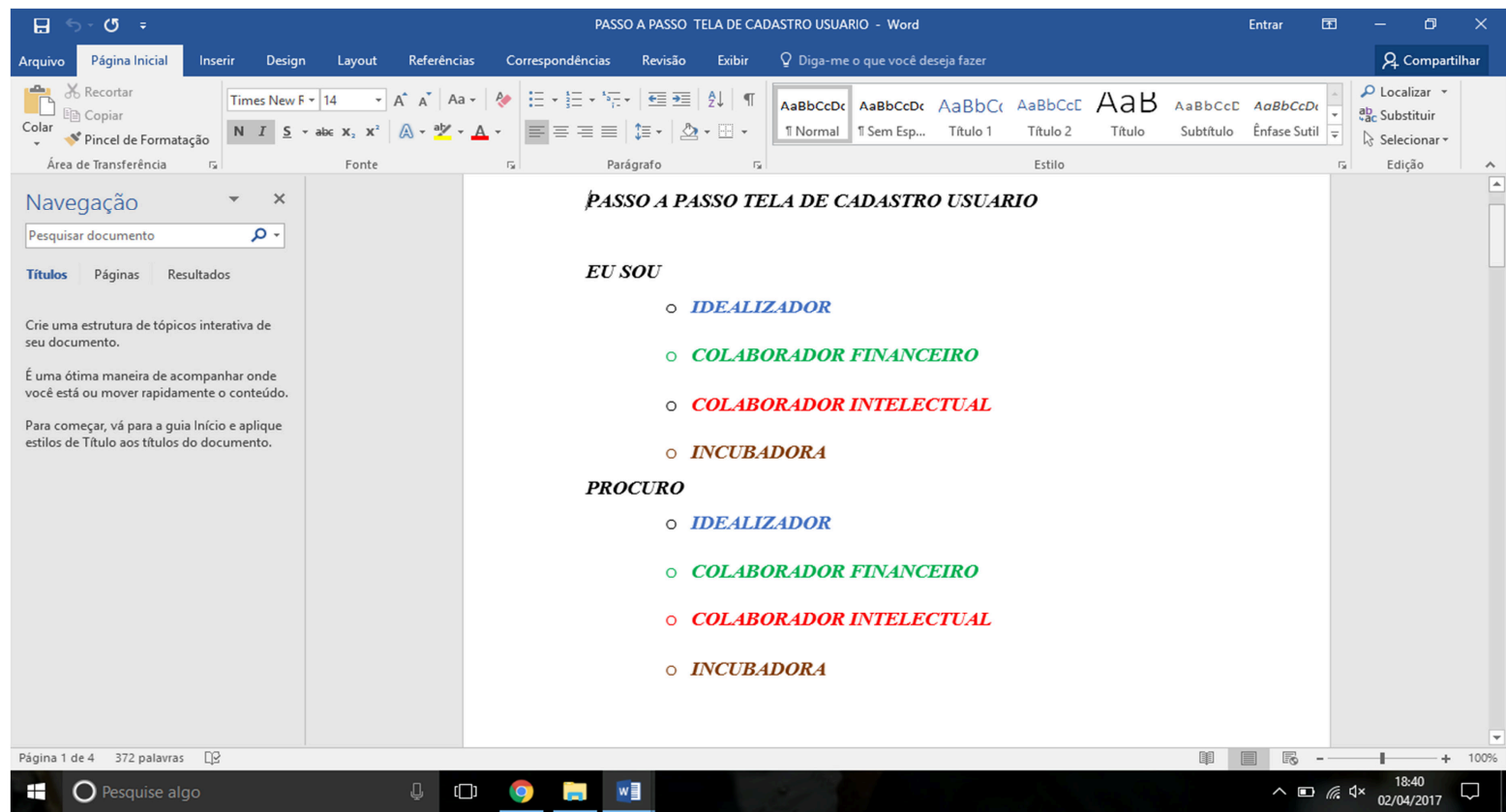

Figura 3: Cadastro perfil específico.

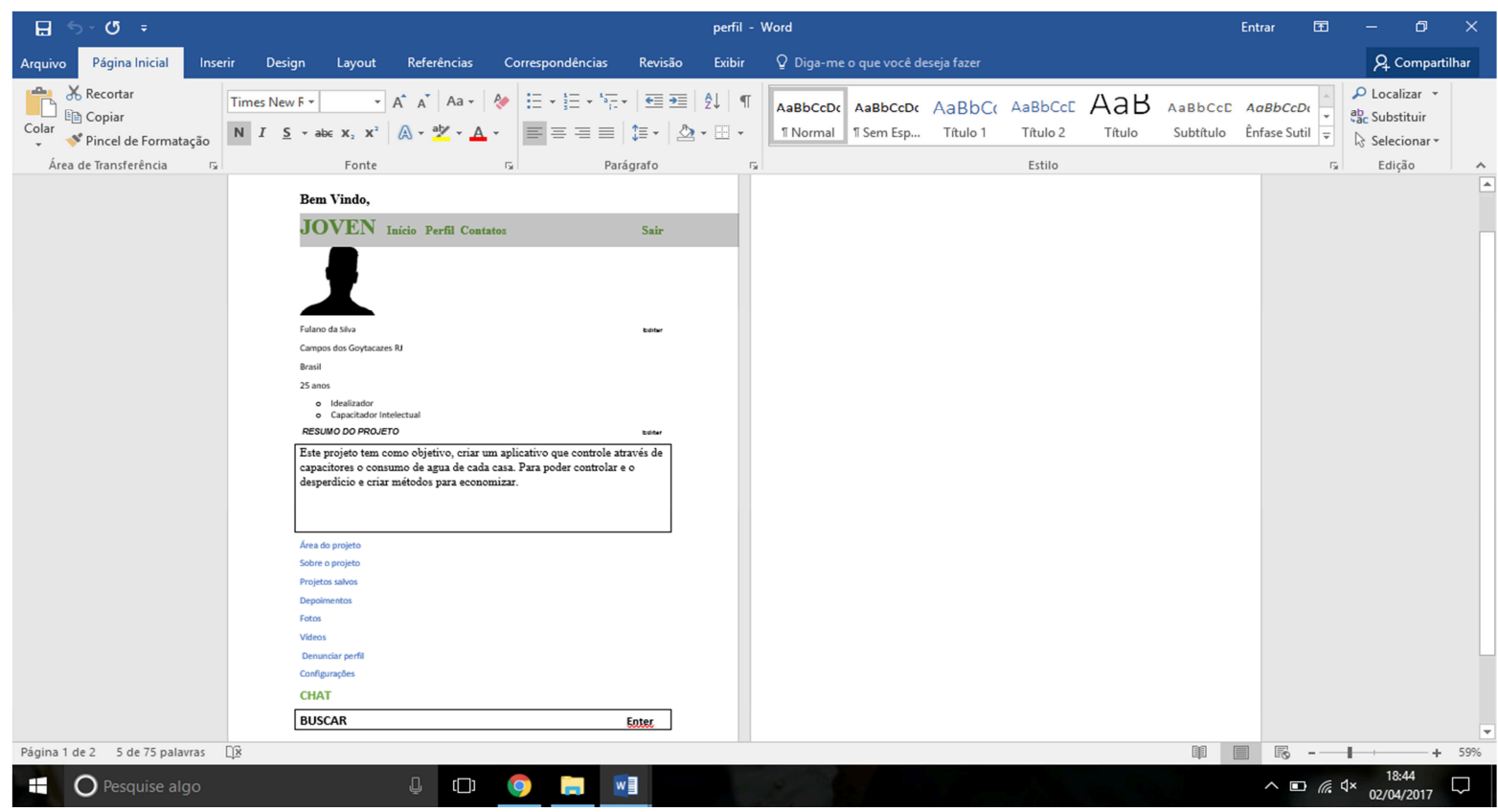

Figura 4: Tela perfil.

\section{CONSIDERAÇÕES}

Referente ao projeto espera-se desenvolver um protótipo de uma Rede Social Digital (RSD), inicialmente voltada para dispositivos móveis, que possibilite conectar e promover diálogo entre atores econômicos (indivíduos que possuam alguma ideia empreendedora e que necessitem do apoio de investidores).

Em relação a benefícios à sociedade, tem-se que a efetividade de um ambiente digital em rede social que permite a interação entre atores econômicos voltados ao empreendedorismo, possibilita um real incentivo ao desenvolvimento local e regional, fomentando a economia e o surgimento de novos negócios. Esta ideia é 
respaldada por Barabási (2002) que afirma ser possível fortalecer as conexões fracas de uma rede e promover a criação de cluster que geram um efeito cascata de crescimento de conexões e fenômenos relacionados a esta rede.

Em relação a proteção da propriedade intelectual, destaca-se que os aprimoramentos tecnológicos decorrentes deste projeto enquadram-se na categoria de autoria de Creative Common e se efetivará pela publicação dos resultados em eventos científicos das áreas correlatas. Ademais há, ainda a possibilidade de registro de patentes por meio do Núcleo de Inovação Tecnológica.

\section{REFERENNCIAS}

CARVALHO, A.M., TOTTI, M.E.F. (orgs.) Formação histórica e econômica do Norte Fluminense. (2006). Rio de Janeiro: Garamond.

IF Fluminense (2014a). Institucional. Campos dos Goytacazes: IF Fluminense. Acessado em: 15/06/16. Disponível em: http://portal.iff.edu.br/institucional.

GRANOVETTER, M. (1973) The strengh of Weak Ties. Chicago: American Journal of Sociology. V.78, N.6, 1360-1380.

MARCUS, (2008)

MARTELETO,R.M, SILVA, A.B.O. (2004) Redes e capital social: o enfoque da informação para o desenvolvimento local. Revista Ciências da Informação. V.33. N. 3. Acessa do em: 19/06/14. Disponível em: http://revista.ibict.br/ciinf/index.php/ciinf/article/view/518.

UEBE MANSUR, A.F. et al (2015) Uma proposta sociométrica para o desenvolvimento do empreendedorismo local. Anais do III Forum Mundial de Educação Profissional e Tecnológica. Recife: IFPE.

UEBE MANSUR,A.F. (2012) Use of Social Networks and Complexity forEnhancement of Academic Learning in Supervised Internships: An Internalization by Doing. Anais da Conferência Internacional sobre Informática na Educação. Porto Alegre: PUC-RS. Acessado em: 14/06/16. Disponível em: www.tise.cl/volumen9/TISE2012/65-72.pdf.

UENF. História da UENF. (2014) Campos dos Goytacazes: Universidade Estadual do Rio de Janeiro. Acessado em: 16/06/16. Disponível em: http://www.uenf.br/portal/index.php/br/historia-da-uenf.html.

ZOOSK. Sites de encontros online. Disponível em: https://www.zoosk.com/lnd/lnd03?exp=br_01. Acesso em: 20 jan.2017.

OLX. Olx- O maior site de classificados do Brasil. Disponível em: <http:/www.olx.com.br>. Acesso em: 02 jan.2017.

GABARDO, Ademir C. Análise de redes sociais. Primeira ed. São Paulo: Novatec editora 1tda, 2015. 141 p.

ANNY, T. E. O; SIMON, F. N Empreendedorismo Sustentável e Stakeholders fornecedores. Revista administração contemporânea: Curitiba, v. 20, n 4, jul./ago..2016. Disponível em http://dx.doi.org/10.1590/1982-7849rac2016150031.Acesso em: 10 ago.2016. 